\title{
Total Syntheses of the Resorcylic Acid Lactone Neocosmosin A and Its Enantiomer
}

\author{
Yiwen Zhang, Michael Dlugosch, Martin Jübermann, Martin G. Banwell,* and Jas S. Ward \\ Research School of Chemistry, Institute of Advanced Studies, The Australian National University, Canberra, ACT 2601, Australia
}

Supporting Information

ABSTRACT: A total synthesis of the structure, 1, assigned to the recently reported resorcylic acid lactone (RAL) neocosmosin A has been established. Olefin-cross metathesis, ring-closing metathesis, palladium-catalyzed Meinwald rearrangement, and Mitsunobu esterification reactions were used as key steps. A late-stage and simple modification to the reaction sequence also provided compound ent-1 that, in fact, represents the true structure of the natural product.

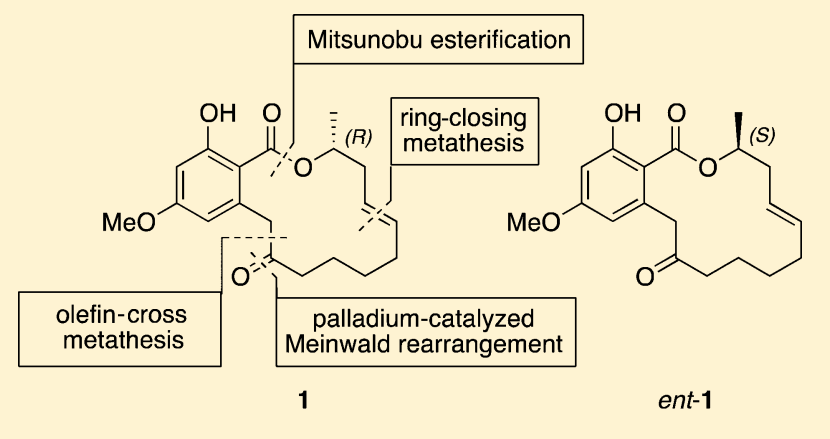

the first time, that some RALs may be useful for modulating pain. Prompted by such observations, the new structures of the neocosmosins, and our previous work ${ }^{3}$ on the assembly of RALs, we commenced synthetic studies in the area. Our initial focus was the preparation of the structure, 1, assigned to neocosmosin A. Herein, we detail total syntheses of compound 1 and its enantiomer (ent-1), thereby establishing, in fact, that it is the latter structure that corresponds to the natural product neocosmosin A. During the course of the studies detailed here, Das and co-workers reported ${ }^{4}$ a distinct synthesis of compound ent-1.

The proven effectiveness of ring-closing metatheses (RCMs) as a means for assembling the 14-membered macrolide of the RALs $^{3 \mathrm{~b}, 5}$ prompted us to pursue this approach to targets $\mathbf{1}$ and ent-1. We also sought to use some of the same building blocks as employed in our earlier studies. ${ }^{3}$ A further consideration was the desire to delay as long as possible the establishment of the single stereogenic center (C2) associated with the target compounds in order that the syntheses (of the two enantiomers) would only diverge at a very late stage.

The opening phase of the syntheses are shown in Scheme 1 and involved an olefin-cross metathesis (OCM) of the previously reported and readily accessible resorcylic acid derivative $4^{3 \mathrm{c}}$ with the known ${ }^{6}$ unsaturated acetal 5 that is readily generated from cyclohexene, as detailed in the Experimental Section. The almost exclusively E-configured alkene 6 (72\%) formed by such means was treated with freshly prepared dimethyldioxirane ${ }^{7}$ to give epoxide 7 (quant.) that, upon exposure to $\mathrm{Pd}(\mathrm{OAc})_{2}$ and $n-\mathrm{Bu}_{3} \mathrm{P}$, engaged in a Meinwald-type rearrangement reaction ${ }^{8}$ to give ketone 8 (88\%). Hydrolysis of the acetal residue within the last compound was achieved by treating its THF/water solution

Received: March 16, 2015

Published: April 1, 2015 
Scheme 1

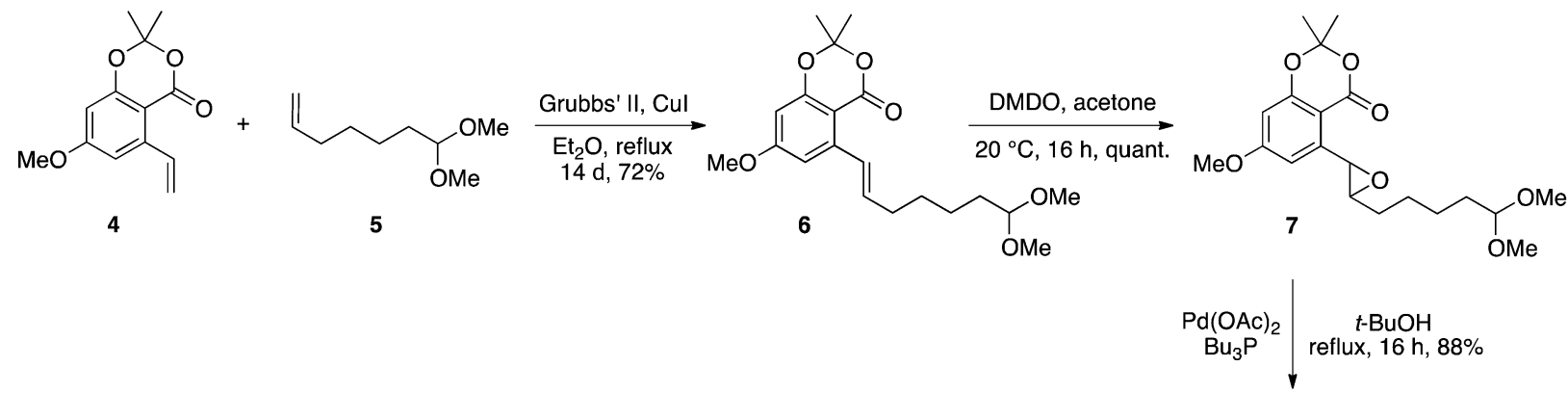

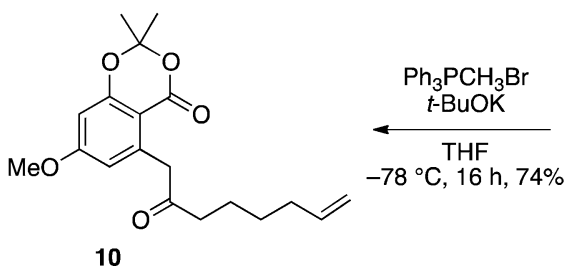<smiles>COc1cc(CC(=O)CCCCC=O)c2c(c1)OC(C)(C)OC2=O</smiles><smiles>COc1cc(CC(=O)CCCCC(OC)OC)c2c(c1)OC(C)(C)OC2=O</smiles>

with pyridium $p$-toluenesulfonate (PPTS) at $50{ }^{\circ} \mathrm{C}$ for $16 \mathrm{~h}$. The resulting keto-aldehyde 9 (89\%) could be selectively methylenated using $1.2 \mathrm{~mol}$ equivalents of the Wittig reagent and so affording the terminal olefin 10 in $74 \%$ yield.

On the basis of our earlier work, ${ }^{3 c}$ we anticipated (Scheme 2) that reaction of compound $\mathbf{1 0}$ with the $R$-configured and commercially available unsaturated alcohol $\mathbf{1 1}$ in the presence sodium hydride would deliver diene 12, the substrate required for the foreshadowed RCM reaction that should complete the synthesis of target 1 . In the event, however, a facile lactonization of compound $\mathbf{1 0}$ took place instead. Thus,

Scheme 2

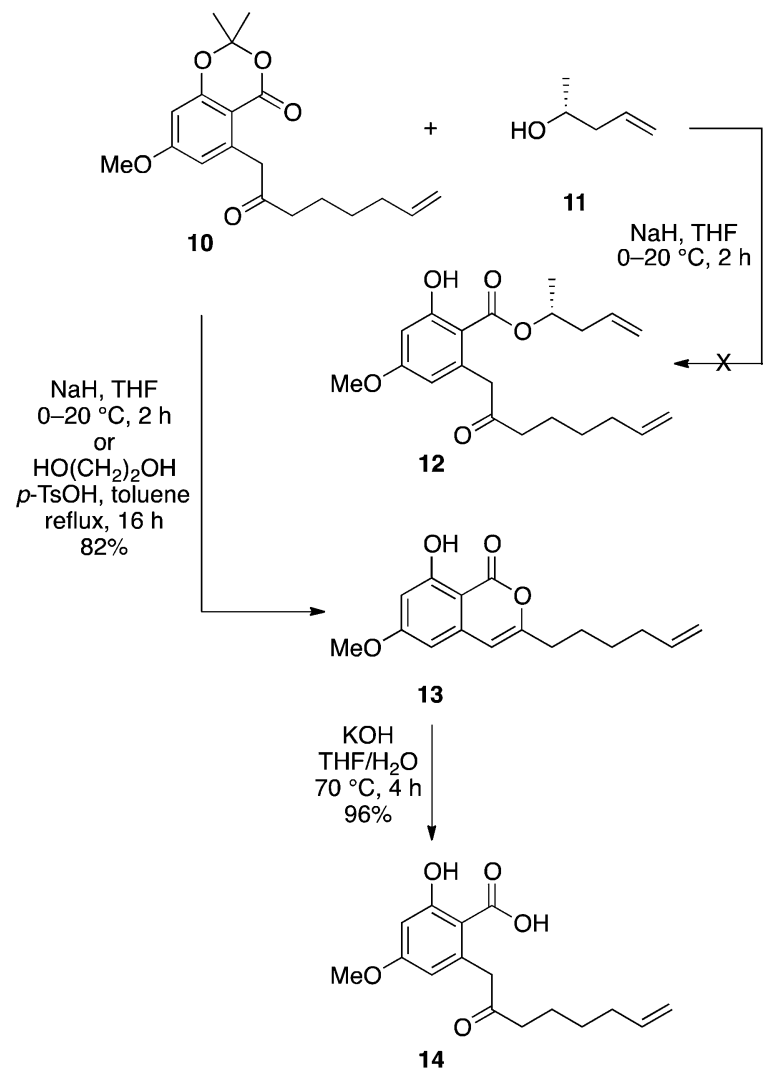

when a mixture of compounds $\mathbf{1 0}$ and $\mathbf{1 1}$ was treated with sodium hydride and subjected to an acidic work up, then a chromatographically separable mixture of lactone 13 (15\%) and the corresponding hydrolysis product 14 (35\%) was obtained. When compound $\mathbf{1 0}$ alone was treated with ethylene glycol and catalytic quantities of $p$-toluenesulfonic acid $(p-\mathrm{TsOH})$ in refluxing toluene, then lactone $\mathbf{1 3}$ could be obtained as the exclusive product of the reaction in $82 \%$ yield. Furthermore, treatment of the latter compound with potassium hydroxide in THF/water afforded, after an acidic workup, the benzoic acid $14(96 \%)$.

The lack of success forming diene $\mathbf{1 2}$ by the pathway detailed above could be circumvented by subjecting commercially available $(S)-(+)-4-p e n t e n-2-o l$ (ent-11) (Scheme 3) to a Mitsunobu reaction using acid $\mathbf{1 4}$ as nucleophile together with a combination of di-isopropyl azodicarboxylate (DIAD) and tri(2-furyl)phosphine $\left[\mathrm{P}(\text { fur })_{3}\right]^{9}$ for activating the alcohol. By such means, the target diene ester 12 was obtained in $78 \%$ yield. This product is assumed to possess the $R$-configuration at

Scheme 3

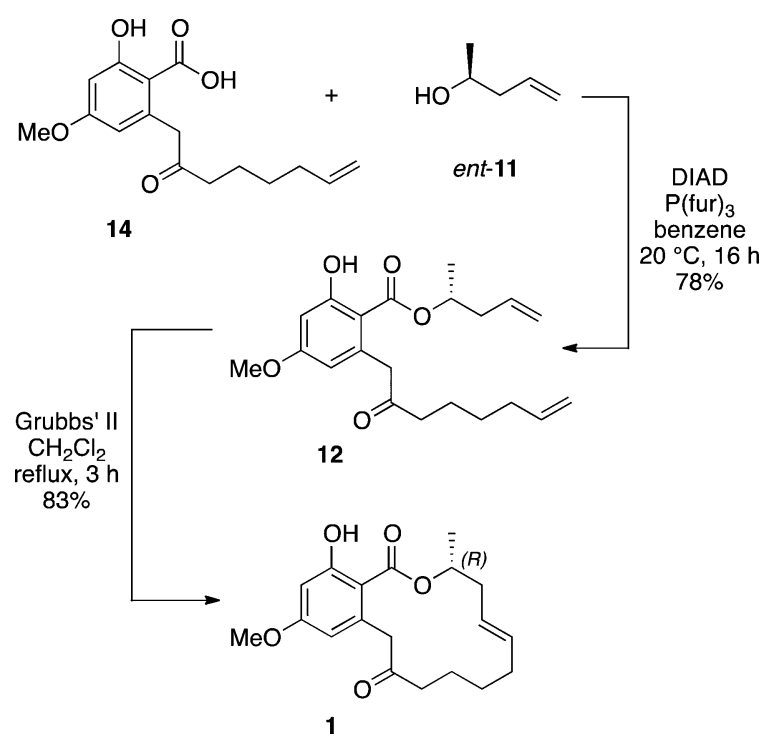

1 
C2 (RAL numbering) by virtue of the operation of the usual $\mathrm{S}_{\mathrm{N}} 2$ pathway in the Mitsunobu reaction. ${ }^{10}$ The pivotal RCM reaction of compound $\mathbf{1 2}$ could be effected using the Grubbs' second generation (Grubbs' II) catalyst ${ }^{11}$ in refluxing dichloromethane, thereby producing crystalline RAL 1 in $83 \%$ yield. The NMR, IR, and MS spectral data obtained on this material matched those reported ${ }^{2 a}$ for neocosmosin A. However, while the specific rotation of compound $\mathbf{1}$ was of a similar magnitude to that reported for the natural product, it was of the opposite sign, thus suggesting that the absolute configuration $(R)$ assigned $^{2 b}$ to this RAL is incorrect. Accordingly, the synthesis of compound ent-1 was pursued.

The synthesis of the macrolide ent-1 was readily achieved by the pathway shown in Scheme 4. This pathway represents a

\section{Scheme 4}

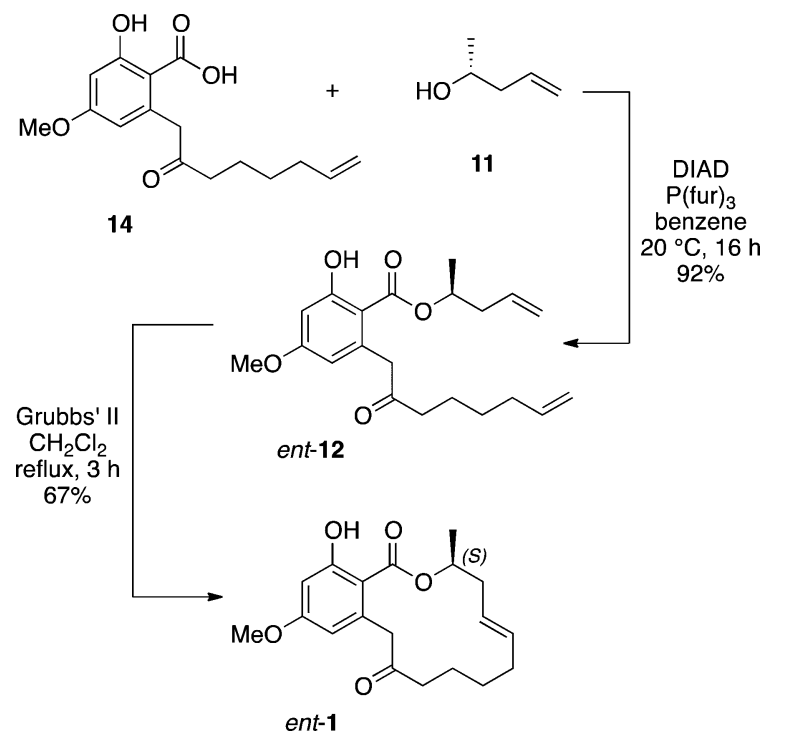

minor modification of the one used to prepare enantiomer $\mathbf{1}$. Thus, ester ent-12 was obtained in $92 \%$ yield by engaging $(R)$ (+)-4-penten-2-ol (11) in a Mitsunobu esterification reaction with benzoic acid 14. The ester was then converted, in $67 \%$ yield, into target ent-1 on exposure to the Grubbs' II catalyst. The assigned structure was in full accord with the derived spectral data, but final confirmation of this (including the illustrated absolute configuration) followed from a single-crystal X-ray analysis, the details of which are provided in the Experimental Section and Supporting Information. As revealed in Table 1, a comparison of the ${ }^{13} \mathrm{C}$ and ${ }^{1} \mathrm{H}$ NMR spectral data derived from compound ent-1 with those reported for neocosmosin A revealed an excellent match. Equally significantly, the specific rotation of the synthetically derived material compared very favorably, in terms of both magnitude and sign, with that reported for the natural product $\left\{[\alpha]_{\mathrm{D}}^{20}-42(c 0.6\right.$, $\left.\mathrm{CHCl}_{3}\right)$ for ent-1 vs $[\alpha]_{\mathrm{D}}^{25}-43\left(c 0.6, \mathrm{CHCl}_{3}\right)$ reported $^{2 \mathrm{a}}$ for neocosmosin $\mathrm{A}$ \}.

The studies detailed above have clearly established that the true structure of the RAL neocosmosin A is represented by ent1 and not 1 as suggested by Cutler et al. ${ }^{2 b, 12}$ The synthetic strategy employed here should be readily adapted to the synthesis of neocosmosins $\mathrm{B}$ and $\mathrm{C}$ as well as a number of related RALs, allowing for detailed studies of the capacity of such compounds to act at human opioid and cannabinoid receptors and thereby enhancing the possibility of identifying new agents for managing pain in mammalian systems.

\section{EXPERIMENTAL SECTION}

General Protocols. Unless otherwise specified, proton $\left({ }^{1} \mathrm{H}\right)$ and carbon $\left({ }^{13} \mathrm{C}\right)$ NMR spectra were recorded at room temperature in base-filtered $\mathrm{CDCl}_{3}$ on a spectrometer operating at $400 \mathrm{MHz}$ for proton and $100 \mathrm{MHz}$ for carbon nuclei. The signal due to residual $\mathrm{CHCl}_{3}$ appearing at $\delta_{\mathrm{H}} 7.26$ and the central resonance of the $\mathrm{CDCl}_{3}$ "triplet" appearing at $\delta_{\mathrm{C}} 77.0$ were used to reference ${ }^{1} \mathrm{H}$ and ${ }^{13} \mathrm{C} \mathrm{NMR}$ spectra, respectively. ${ }^{1} \mathrm{H}$ NMR data are recorded as follows: chemical shift $(\delta)$ [multiplicity, coupling constant $(\mathrm{s}) J(\mathrm{~Hz})$, relative integral], where multiplicity is defined as $\mathrm{s}=$ singlet, $\mathrm{d}=$ doublet, $\mathrm{t}=$ triplet, $\mathrm{q}=$ quartet, $\mathrm{m}=$ multiplet, or combinations of the above. Samples were analyzed by infrared spectroscopy $\left(\nu_{\max }\right)$ as thin films on $\mathrm{KBr}$ plates. Low-resolution ESI mass spectra were recorded on a single quadrupole liquid chromatograph-mass spectrometer, whereas high-resolution measurements were conducted on a time-of-flight instrument. Lowand high-resolution EI mass spectra were recorded on a magneticsector machine. Melting points were measured on an automated melting point system and are uncorrected. Analytical thin-layer chromatography (TLC) was performed on aluminum-backed 0.2 $\mathrm{mm}$ thick silica gel $60 \mathrm{~F}_{254}$ plates. Eluted plates were visualized using a $254 \mathrm{~nm}$ UV lamp and/or by treatment with a suitable dip followed by heating. These dips included phosphomolybdic acid/ceric sulfate/ sulfuric acid (conc.)/water (37.5 g:7.5 g:37.5 g:720 mL) or potassium permanganate/potassium carbonate/5\% sodium hydroxide aqueous solution/water (3 g:20 g:5 mL:300 mL). Flash chromatographic separations were carried out following protocols defined by Still et al. ${ }^{13}$ with silica gel $60(40-63 \mu \mathrm{m})$ as the stationary phase and using the AR- or HPLC-grade solvents indicated. Starting materials and reagents were generally commercially available and were used as supplied. Drying agents and other inorganic salts were purchased from commercial suppliers. Tetrahydrofuran (THF), methanol, and dichloromethane were dried using a solvent purification system that is based upon a technology originally described by Grubbs et al. ${ }^{14}$ Where necessary, reactions were performed under a nitrogen or argon atmosphere.

Compound 5. Step i. Ozone was passed through a magnetically stirred solution of cyclohexene $(15.35 \mathrm{~mL}, 150 \mathrm{mmol})$ in dry dichloromethane $(300 \mathrm{~mL})$ containing dry $\mathrm{MeOH}(100 \mathrm{~mL})$ maintained at $-78{ }^{\circ} \mathrm{C}$ (dry ice/acetone bath). Once the reaction mixture remained blue, nitrogen was bubbled through it until the color was discharged, at which point it was treated with $p-\mathrm{TsOH} \cdot \mathrm{H}_{2} \mathrm{O}(2.90$ $\mathrm{g}, 15 \mathrm{mmol}$ ) and the cooling bath was removed. With continuing stirring, the reaction mixture was allowed to warm to $20^{\circ} \mathrm{C}(\mathrm{ca} .2 \mathrm{~h})$ before it was treated, in portions, with $\mathrm{Na}_{2} \mathrm{CO}_{3}(3.28 \mathrm{~g}, 39 \mathrm{mmol})$ and, after $0.5 \mathrm{~h}$, with $\mathrm{Me}_{2} \mathrm{~S}(24 \mathrm{~mL}, 330 \mathrm{mmol})$ that was added dropwise over $0.5 \mathrm{~h}$. The resulting mixture was stirred at $20{ }^{\circ} \mathrm{C}$ for $16 \mathrm{~h}$ and then quenched with $\mathrm{H}_{2} \mathrm{O}(200 \mathrm{~mL})$. The separated aqueous phase was extracted with diethyl ether $(4 \times 200 \mathrm{~mL})$, and the combined organic phases were dried $\left(\mathrm{MgSO}_{4}\right)$, filtered, and concentrated under reduced pressure. The light-yellow oil thus obtained was purified by flash chromatography (silica, 4:1 v/v hexane/ethyl acetate elution), and after concentration of the appropriate fractions $\left(R_{\mathrm{f}}=0.3\right.$ in $9: 1 \mathrm{v} / \mathrm{v}$ hexane/ethyl acetate), 6,6-dimethoxyhexanal ${ }^{6}$ (22.93 g, 95\%) was obtained as a clear, colorless oil. ${ }^{1} \mathrm{H}$ NMR $\left(400 \mathrm{MHz}, \mathrm{CDCl}_{3}\right) \delta 9.74$ (s, $1 \mathrm{H}), 4.35-4.32$ (complex m, 1H), 3.29 (s, 6H), 2.45-2.40 (complex m, 2H), 1.68-1.57 (complex m, 4H), 1.40-1.33 (complex $\mathrm{m}, 2 \mathrm{H}) ;{ }^{13} \mathrm{C} \mathrm{NMR}\left(100 \mathrm{MHz}, \mathrm{CDCl}_{3}\right) \delta 202.5,104.4,52.9,43.9,32.4$, 24.3, 22.0; IR $\nu_{\max } 3424,2947,2831,2721,1726,1460,1388,1366$, $1192,1128,1074,1052,958 \mathrm{~cm}^{-1}$; MS (ESI, +ve) $\mathrm{m} / z 199\left[(\mathrm{M}+\mathrm{K})^{+}\right.$, $100 \%], 183\left[(\mathrm{M}+\mathrm{Na})^{+}, 71\right]$; HRMS $(\mathrm{M}+\mathrm{Na})^{+}$calcd for $\mathrm{C}_{8} \mathrm{H}_{16} \mathrm{NaO}_{3}, 183.0997$; found, 183.0997 .

Step ii. A magnetically stirred suspension of $\mathrm{MePPh}_{3} \mathrm{Br}(61.09 \mathrm{~g}$, $167.60 \mathrm{mmol})$ in dry THF $(200 \mathrm{~mL})$ maintained under nitrogen at 0 ${ }^{\circ} \mathrm{C}$ (ice bath) was treated, dropwise over $0.5 \mathrm{~h}$, with $n$-BuLi $(104.7 \mathrm{~mL}$ of a $1.6 \mathrm{M}$ solution in hexanes, $167.6 \mathrm{mmol}$ ). The ensuing orange suspension was stirred at $0{ }^{\circ} \mathrm{C}$ for $0.5 \mathrm{~h}$ before a solution of 6,6 - 
Table 1. Comparison of the ${ }^{13} \mathrm{C}$ and ${ }^{1} \mathrm{H}$ NMR Data Recorded for Synthetically Derived Compound ent-1 with Those Reported for Neocosmosin A

\begin{tabular}{|c|c|c|c|}
\hline \multicolumn{2}{|c|}{${ }^{13} \mathrm{C} \operatorname{NMR}\left(\delta_{\mathrm{C}}\right)$} & \multicolumn{2}{|c|}{${ }^{1} \mathrm{H} \operatorname{NMR}\left(\delta_{\mathrm{H}}\right)$} \\
\hline neocosmosin $A^{a}$ & compound ent-1 ${ }^{b}$ & neocosmosin $A^{c}$ & compound ent-1 ${ }^{d}$ \\
\hline 208.2 & 208.4 & $11.98, \mathrm{~s}, 1 \mathrm{H}$ & $11.98, \mathrm{~s}, 1 \mathrm{H}$ \\
\hline 170.7 & 170.8 & $6.44, \mathrm{~d}, J=2.5 \mathrm{~Hz}, 1 \mathrm{H}$ & $6.42, \mathrm{~d}, J=4.0 \mathrm{~Hz}, 1 \mathrm{H}$ \\
\hline 166.0 & 166.1 & $6.24, \mathrm{~d}, J=2.5 \mathrm{~Hz}, 1 \mathrm{H}$ & $6.21, \mathrm{~d}, J=4.0 \mathrm{~Hz}, 1 \mathrm{H}$ \\
\hline 163.9 & 164.0 & $5.51, \mathrm{~m}, 1 \mathrm{H}$ & $5.50-5.38$, complex $\mathrm{m}, 2 \mathrm{H}$ \\
\hline 139.1 & 139.2 & $5.46, \mathrm{~m}, 1 \mathrm{H}$ & \\
\hline 135.1 & 135.3 & $5.35, \mathrm{~m}, 1 \mathrm{H}$ & $5.32, \mathrm{~m}, 1 \mathrm{H}$ \\
\hline 124.5 & 124.6 & $4.42, \mathrm{~d}, J=16.8 \mathrm{~Hz}, 1 \mathrm{H}$ & $4.38, \mathrm{~d}, J=16.7 \mathrm{~Hz}, 1 \mathrm{H}$ \\
\hline 112.1 & 112.2 & $3.82, \mathrm{~s}, 3 \mathrm{H}$ & $3.79, \mathrm{~s}, 3 \mathrm{H}$ \\
\hline 105.7 & 105.8 & $3.51, \mathrm{~d}, J=16.8 \mathrm{~Hz}, 1 \mathrm{H}$ & $3.48, \mathrm{~d}, J=16.7 \mathrm{~Hz}, 1 \mathrm{H}$ \\
\hline 100.1 & 100.2 & $2.63, \mathrm{~m}, 1 \mathrm{H}$ & $2.59, \mathrm{~m}, 1 \mathrm{H}$ \\
\hline 72.9 & 73.0 & $2.55, \mathrm{~m}, 1 \mathrm{H}$ & $2.52, \mathrm{~m}, 1 \mathrm{H}$ \\
\hline 55.4 & 55.5 & 2.39, m, 1H & 2.36, $\mathrm{m}, 1 \mathrm{H}$ \\
\hline 50.2 & 50.4 & $2.27, \mathrm{~m}, 1 \mathrm{H}$ & $2.25, \mathrm{~m}, 1 \mathrm{H}$ \\
\hline 40.8 & 40.9 & $2.09, \mathrm{~m}, 2 \mathrm{H}$ & $2.14-2.00$, complex $\mathrm{m}, 2 \mathrm{H}$ \\
\hline 37.7 & 37.8 & $1.65, \mathrm{~m}, 2 \mathrm{H}$ & $1.69-1.40$, complex $\mathrm{m}, 4 \mathrm{H}$ \\
\hline 32.7 & 32.9 & $1.63, \mathrm{~m}, 1 \mathrm{H}$ & \\
\hline 25.2 & 25.3 & $1.42, \mathrm{~m}, 1 \mathrm{H}$ & \\
\hline 22.1 & 22.2 & $1.40, \mathrm{~d}, J=6.5 \mathrm{~Hz}, 3 \mathrm{H}$ & $1.37, \mathrm{~d}, J=6.5 \mathrm{~Hz}, 3 \mathrm{H}$ \\
\hline 18.9 & 19.0 & & \\
\hline
\end{tabular}

${ }^{a}$ Data obtained from ref 2a; recorded in $\mathrm{CDCl}_{3}$ at $150 \mathrm{MHz} .{ }^{b}$ Data recorded in $\mathrm{CDCl}_{3}$ at $100 \mathrm{MHz} \cdot{ }^{c}$ Data obtained from ref $2 \mathrm{a}$; recorded in $\mathrm{CDCl}_{3}$ at $500 \mathrm{MHz} .{ }^{d}$ Data recorded in $\mathrm{CDCl}_{3}$ at $400 \mathrm{MHz}$.

dimethoxyhexanal $(13.43 \mathrm{~g}, 83.80 \mathrm{mmol})$ in dry THF $(50 \mathrm{~mL})$ was added dropwise. The resulting yellow mixture was left to warm to 20 ${ }^{\circ} \mathrm{C}$ over $16 \mathrm{~h}$ and was then treated with $\mathrm{NH}_{4} \mathrm{Cl}(200 \mathrm{~mL}$ of a saturated aqueous solution). The separated aqueous phase was extracted with diethyl ether $(2 \times 200 \mathrm{~mL})$, and the combined organic phases were washed with brine $(1 \times 200 \mathrm{~mL})$ before being dried $\left(\mathrm{MgSO}_{4}\right)$, filtered, and then concentrated under reduced pressure. The yellow oil thus obtained was subjected to flash chromatography (silica, 50:1 v/v hexane/ethyl acetate elution), and concentration of the relevant fractions $\left(R_{f}=0.5\right.$ in $9: 1 \mathrm{v} / \mathrm{v}$ hexane/ethyl acetate $)$ afforded compound $5^{6}(7.45 \mathrm{~g}, 56 \%)$ as a clear, colorless oil. ${ }^{1} \mathrm{H}$ NMR $(400$ $\left.\mathrm{MHz}, \mathrm{CDCl}_{3}\right) \delta 5.79(\mathrm{~m}, 1 \mathrm{H}), 5.00-4.91$ (complex m, 2H), $4.35(\mathrm{t}, J$ $=5.8 \mathrm{~Hz}, 1 \mathrm{H}), 3.30(\mathrm{~s}, 6 \mathrm{H}), 2.04(\mathrm{~m}, 2 \mathrm{H}), 1.59(\mathrm{~m}, 2 \mathrm{H}), 1.43-1.30$ (complex m, 4H); ${ }^{13} \mathrm{C}$ NMR $\left(100 \mathrm{MHz}, \mathrm{CDCl}_{3}\right) \delta 138.9,114.5,104.6$, 52.7, 33.8, 32.5, 28.9, 24.2; IR $\nu_{\max } 3077,2978,2929,2859,2830$, 1641, 1462, 1416, 1385, 1363, 1191, 1127, 1077, 1054, $910 \mathrm{~cm}^{-1}$; MS $(\mathrm{ESI},+\mathrm{ve}) \mathrm{m} / z 171\left[(\mathrm{M}+\mathrm{Na})^{+}, 100 \%\right], 159\left[(\mathrm{M}+\mathrm{H})^{+}, 27\right]$; HRMS $(\mathrm{M}+\mathrm{Na})^{+}$calcd for $\mathrm{C}_{9} \mathrm{H}_{18} \mathrm{NaO}_{2}, 181.1204$; found, 181.1205.

Compound 6. A magnetically stirred and degassed mixture of compound $4^{3 \mathrm{c}}(1.25 \mathrm{~g}, 5.14 \mathrm{mmol})$, compound $5(1.02 \mathrm{~g}, 6.43 \mathrm{mmol})$, Grubbs' II catalyst (110 mg, $0.13 \mathrm{mmol})$, and CuI ( $30 \mathrm{mg}, 0.15 \mathrm{mmol})$ in dry $\mathrm{Et}_{2} \mathrm{O}(25 \mathrm{~mL})$ was stirred at reflux under an atmosphere of argon. Grubbs' II catalyst $(110 \mathrm{mg}, 0.13 \mathrm{mmol})$ and $\mathrm{CuI}(30 \mathrm{mg}, 0.15$ mmol) were each added seven times every other day, and olefin 5 $(1.02 \mathrm{~g}, 6.43 \mathrm{mmol})$ every third day and three times in total. After 14 days, the reaction mixture was concentrated under reduced pressure, and the residue thus obtained subjected to flash chromatography (silica, $8: 1 \mathrm{v} / \mathrm{v}$ hexane/ethyl acetate elution) to afford compound 6 $(1.35 \mathrm{~g}, 72 \%)$ as a clear, light-yellow oil. ${ }^{1} \mathrm{H}$ NMR $\left(400 \mathrm{MHz}, \mathrm{CDCl}_{3}\right)$ $\delta 7.39(\mathrm{~d}, J=15.7 \mathrm{~Hz}, 1 \mathrm{H}), 6.69(\mathrm{~d}, J=2.6 \mathrm{~Hz}, 1 \mathrm{H}), 6.27(\mathrm{~d}, J=2.6$ $\mathrm{Hz}, 1 \mathrm{H}), 6.14(\mathrm{dt}, J=15.7$ and $6.9 \mathrm{~Hz}, 1 \mathrm{H}), 4.32(\mathrm{t}, J=5.7 \mathrm{~Hz}, 1 \mathrm{H})$, $3.79(\mathrm{~s}, 3 \mathrm{H}), 3.26(\mathrm{~s}, 6 \mathrm{H}), 2.23(\mathrm{~m}, 2 \mathrm{H}), 1.64(\mathrm{~s}, 6 \mathrm{H}), 1.58(\mathrm{~m}, 2 \mathrm{H})$, $1.48(\mathrm{~m}, 2 \mathrm{H}), 1.38(\mathrm{~m}, 2 \mathrm{H}) ;{ }^{13} \mathrm{C}$ NMR $\left(100 \mathrm{MHz}, \mathrm{CDCl}_{3}\right) \delta 164.7$, 160.2 , 158.7, 144.2, 135.0, 128.3, 108.1, 104.9, 104.5, 103.7, 100.1, 55.6, 52.7, 33.0, 32.4, 29.0, 25.6, 24.2; IR $\nu_{\max } 2992,2942,2857,1729$, $1605,1573,1430,1389,1275,1204,1161,1128,1072,968,914,855$ $\mathrm{cm}^{-1}$; MS (ESI, +ve) $\mathrm{m} / z 387\left[(\mathrm{M}+\mathrm{Na})^{+}, 100 \%\right]$; HRMS $(\mathrm{M}+\mathrm{Na})^{+}$ calcd for $\mathrm{C}_{20} \mathrm{H}_{28} \mathrm{NaO}_{6}$, 387.1784; found, 387.1785.

Efforts to accelerate this sluggish reaction using different solvents and/or microwave irradiation conditions were all unsuccessful.
Compound 7. A magnetically stirred solution of olefin $6(428 \mathrm{mg}$, $1.17 \mathrm{mmol}$ ) in acetone $(3 \mathrm{~mL})$ maintained at $0{ }^{\circ} \mathrm{C}$ (ice-bath) was treated with dimethyldioxirane ${ }^{7}(26 \mathrm{~mL}$ of a $67 \mathrm{mM}$ solution in acetone, $1.76 \mathrm{mmol}$ ). The resulting yellow solution was stirred at 20 ${ }^{\circ} \mathrm{C}$ for $16 \mathrm{~h}$ and then concentrated under reduced pressure to afford compound 7 (445 mg, quant.) as a clear, light-yellow oil. ${ }^{1} \mathrm{H}$ NMR $\left(400 \mathrm{MHz}, \mathrm{CDCl}_{3}\right) \delta 6.66(\mathrm{~d}, J=2.6 \mathrm{~Hz}, 1 \mathrm{H}), 6.32(\mathrm{~d}, J=2.6 \mathrm{~Hz}$, $1 \mathrm{H}), 4.40(\mathrm{~d}, J=2.2 \mathrm{~Hz}, 1 \mathrm{H}), 4.33(\mathrm{~m}, 1 \mathrm{H}), 3.80(\mathrm{~s}, 3 \mathrm{H}), 3.27(\mathrm{~s}$, $6 \mathrm{H}), 2.71(\mathrm{~m}, 1 \mathrm{H}), 1.69(\mathrm{~s}, 3 \mathrm{H}), 1.66(\mathrm{~s}, 3 \mathrm{H}), 1.64-1.49($ complex m, $6 \mathrm{H}), 1.39(\mathrm{~m}, 2 \mathrm{H}) ;{ }^{13} \mathrm{C} \mathrm{NMR}\left(100 \mathrm{MHz}, \mathrm{CDCl}_{3}\right) \delta 165.9,160.1$, $158.5,145.0,129.2$, 106.0, 105.5, 104.5, 104.3, 100.9, 63.4, 57.1, 55.7, 52.6, 32.4, 32.3, 26.4, 25.5, 24.8, 24.5; IR $\nu_{\max } 2944,2862,1726,1670$, $1612,1582,1442,1378,1283,1203,1160,1131,1068,963,913,854$, $751 \mathrm{~cm}^{-1}$; MS (ESI, +ve) $\mathrm{m} / z 403\left[(\mathrm{M}+\mathrm{Na})^{+}, 100 \%\right], 381[(\mathrm{M}+$ $\mathrm{H})^{+}$, 4]; HRMS $(\mathrm{M}+\mathrm{Na})^{+}$calcd for $\mathrm{C}_{20} \mathrm{H}_{28} \mathrm{NaO}_{7}, 403.1733$; found, 403.1733 .

Compound 8. A magnetically stirred solution of compound 7 $(1.30 \mathrm{~g}, 3.41 \mathrm{mmol})$ and $\mathrm{Pd}(\mathrm{OAc})_{2}$ in degassed $t-\mathrm{BuOH}(60 \mathrm{~mL})$ maintained at $20^{\circ} \mathrm{C}$ under nitrogen was treated with $n$ - $\mathrm{Bu}_{3} \mathrm{P}(860 \mu \mathrm{L}$, $3.41 \mathrm{mmol}$ ), and the ensuing orange solution was heated at reflux for $16 \mathrm{~h}$. The cooled reaction mixture was concentrated under reduced pressure, and the residue was subjected to flash chromatography (silica, $3: 1 \mathrm{v} / \mathrm{v}$ hexane/ethyl acetate elution) to afford, after concentration of the appropriate fractions $\left(R_{f}=0.2\right.$ in $4: 1 \mathrm{v} / \mathrm{v}$ hexane/ethyl acetate), compound $8(1.15 \mathrm{~g}, 88 \%)$ as a clear, lightyellow oil. ${ }^{1} \mathrm{H} \mathrm{NMR}\left(400 \mathrm{MHz}, \mathrm{CDCl}_{3}\right) \delta 6.36(\mathrm{~d}, J=2.5 \mathrm{~Hz}, 1 \mathrm{H})$, $6.34(\mathrm{~d}, J=2.5 \mathrm{~Hz}, 1 \mathrm{H}), 4.32(\mathrm{t}, J=5.7 \mathrm{~Hz}, 1 \mathrm{H}), 4.07(\mathrm{~s}, 2 \mathrm{H}), 3.79(\mathrm{~s}$, $3 \mathrm{H}), 3.28(\mathrm{~s}, 6 \mathrm{H}), 2.58(\mathrm{t}, J=7.4 \mathrm{~Hz}, 2 \mathrm{H}), 1.67(\mathrm{~s}, 6 \mathrm{H}), 1.64-1.55$ (complex m, 4H), $1.34(\mathrm{~m}, 2 \mathrm{H}) ;{ }^{13} \mathrm{C}$ NMR $\left(100 \mathrm{MHz} \mathrm{CDCl}_{3}\right) \delta$ 206.9, 164.9, 160.8, 159.0, 140.8, 114.1, 105.4, 104.4, 100.2, 55.6, 52.7, 48.6, 42.7, 32.3, 25.6, 24.2, 23.4 (one signal obscured or overlapping); IR $\nu_{\max } 2989,2945,2831,1724,1614,1581,1436,1286,1205,1161$, $1127,1067 \mathrm{~cm}^{-1}$; MS (ESI, +ve) $\mathrm{m} / z 403\left[(\mathrm{M}+\mathrm{Na})^{+}, 100 \%\right]$; HRMS $(\mathrm{M}+\mathrm{Na})^{+}$calcd for $\mathrm{C}_{20} \mathrm{H}_{28} \mathrm{NaO}_{7}, 403.1733$; found, 403.1734.

Compound 9. A magnetically stirred solution of compound 8 (55 $\mathrm{mg}, 0.14 \mathrm{mmol})$ in $\mathrm{THF} /$ water $(6 \mathrm{~mL}$ of a $2: 1 \mathrm{v} / \mathrm{v}$ mixture $)$ was treated with PPTS $(18 \mathrm{mg}, 0.07 \mathrm{mmol})$, and the ensuing mixture was stirred at $60{ }^{\circ} \mathrm{C}$ for $16 \mathrm{~h}$. The cooled reaction mixture was concentrated under reduced pressure, the residue was diluted with water $(50 \mathrm{~mL})$, and the ensuing mixture was extracted with 
dichloromethane $(3 \times 30 \mathrm{~mL})$. The combined organic phases were washed with brine $(1 \times 50 \mathrm{~mL})$ before being dried $\left(\mathrm{Na}_{2} \mathrm{SO}_{4}\right)$, filtered, and then concentrated under reduced pressure. The residue was purified by flash chromatography (silica, $3: 1 \mathrm{v} / \mathrm{v}$ hexane/ethyl acetate elution), and concentration of the relevant fractions $\left(R_{f}=0.4\right.$ in $1: 1 \mathrm{v} /$ $\mathrm{v}$ hexane/ethyl acetate) afforded compound $9(41 \mathrm{mg}, 89 \%)$ as a clear, colorless oil. ${ }^{1} \mathrm{H}$ NMR $\left(400 \mathrm{MHz}, \mathrm{CDCl}_{3}\right) \delta 9.74(\mathrm{~s}, 1 \mathrm{H}), 6.38(\mathrm{~d}, J=$ $2.2 \mathrm{~Hz}, 1 \mathrm{H}), 6.36(\mathrm{~d}, J=2.2 \mathrm{~Hz}, 1 \mathrm{H}), 4.07(\mathrm{~s}, 2 \mathrm{H}), 3.81(\mathrm{~s}, 3 \mathrm{H}), 2.63$ (m, 2H), $2.44(\mathrm{~m}, 2 \mathrm{H}), 1.69(\mathrm{~s}, 6 \mathrm{H}), 1.65(\mathrm{~m}, 4 \mathrm{H}) ;{ }^{13} \mathrm{C}$ NMR $(100$ $\left.\mathrm{MHz} \mathrm{CDCl}_{3}\right) \delta 206.5,202.5,165.0,160.8,159.0,140.6,114.2,105.5$, 105.3, 100.3, 55.6, 48.7, 43.7, 42.3, 25.6, 23.0, 21.6; IR $\nu_{\text {max }} 2997,2944$, $2725,1721,1614,1581,1436,1286,1205,1161,1067 \mathrm{~cm}^{-1}$; MS (ESI, $+\mathrm{ve}) \mathrm{m} / z 373\left[(\mathrm{M}+\mathrm{K})^{+}, 96 \%\right], 357\left[(\mathrm{M}+\mathrm{Na})^{+}, 100\right], 335[(\mathrm{M}+$ $\left.\mathrm{H})^{+}, 52\right]$; HRMS $(\mathrm{M}+\mathrm{Na})^{+}$calcd for $\mathrm{C}_{18} \mathrm{H}_{22} \mathrm{NaO}_{6}, 357.1314$; found, 357.1306 .

Compound 10. A magnetically stirred suspension of $\mathrm{MePPh}_{3} \mathrm{Br}$ $(950 \mathrm{mg}, 2.61 \mathrm{mmol})$ in dry THF $(10 \mathrm{~mL})$ maintained at $0{ }^{\circ} \mathrm{C}$ under nitrogen was treated with $t$-BuOK $(2.5 \mathrm{~mL}$ of a $1.0 \mathrm{M}$ solution in THF, $2.50 \mathrm{mmol}$ ), and the ensuing yellow suspension was stirred at 0 ${ }^{\circ} \mathrm{C}$ for $0.5 \mathrm{~h}$ and then added to a magnetically stirred solution of compound $9(726 \mathrm{mg}, 2.17 \mathrm{mmol})$ in dry THF $(15 \mathrm{~mL})$ maintained at $-78^{\circ} \mathrm{C}$ under a nitrogen atmosphere. The resulting yellow suspension was stirred at $-78{ }^{\circ} \mathrm{C}$ for $0.5 \mathrm{~h}$ and then $0{ }^{\circ} \mathrm{C}$ for $5 \mathrm{~h}$ before being treated, successively, with $\mathrm{NH}_{4} \mathrm{Cl}(10 \mathrm{~mL}$ of a saturated aqueous solution $)$ and water $(20 \mathrm{~mL})$ and then extracted with $\mathrm{Et}_{2} \mathrm{O}(3 \times 30$ $\mathrm{mL})$. The combined organic phases were dried $\left(\mathrm{MgSO}_{4}\right)$, filtered, and then concentrated under reduced pressure, and the residue was subjected to flash chromatography (silica, 6:1 v/v hexane/ethyl acetate elution). Concentration of the appropriate fractions $\left(R_{f}=0.3\right.$ in $4: 1 \mathrm{v} /$ $\mathrm{v}$ hexane/ethyl acetate) gave olefin $10(533 \mathrm{mg}, 74 \%)$ as a clear, colorless oil. ${ }^{1} \mathrm{H}$ NMR $\left(400 \mathrm{MHz}, \mathrm{CDCl}_{3}\right) \delta 6.36(\mathrm{~d}, J=2.5 \mathrm{~Hz}, 1 \mathrm{H})$, $6.33(\mathrm{~d}, J=2.5 \mathrm{~Hz}, 1 \mathrm{H}), 5.76(\mathrm{~m}, 1 \mathrm{H}), 4.99-4.88($ complex m, $2 \mathrm{H})$, $4.07(\mathrm{~s}, 2 \mathrm{H}), 3.78(\mathrm{~s}, 3 \mathrm{H}), 2.57(\mathrm{t}, J=7.4 \mathrm{~Hz}, 2 \mathrm{H}), 2.03(\mathrm{~m}, 2 \mathrm{H}), 1.67$ $(\mathrm{s}, 6 \mathrm{H}), 1.61(\mathrm{~m}, 2 \mathrm{H}), 1.38(\mathrm{~m}, 2 \mathrm{H}) ;{ }^{13} \mathrm{C}$ NMR $\left(100 \mathrm{MHz} \mathrm{CDCl}_{3}\right) \delta$ $207.0,164.9,160.8,159.0,140.8,138.6,114.5,114.1,105.4,100.2$, 55.6, 48.6, 42.6, 33.6, 28.4, 25.6, 23.1 (signal due to one carbon obscured or overlapping); IR $\nu_{\max } 2940,1724,1614,1581,1436,1285$, 1205, 1160, 1067, $911 \mathrm{~cm}^{-1}$; MS (ESI, +ve) $\mathrm{m} / z 355\left[(\mathrm{M}+\mathrm{Na})^{+}\right.$, $100 \%], 333\left[(\mathrm{M}+\mathrm{H})^{+}, 34\right]$; HRMS $(\mathrm{M}+\mathrm{Na})^{+}$calcd for $\mathrm{C}_{19} \mathrm{H}_{24} \mathrm{NaO}_{5}$, 355.1521; found, 355.1521 .

Compound 13. Method A. A magnetically stirred solution of compounds $10(164 \mathrm{mg}, 0.49 \mathrm{mmol})$ and $11(67 \mathrm{mg}, 0.74 \mathrm{mmol})$ in dry THF $(3 \mathrm{~mL})$ maintained at $0{ }^{\circ} \mathrm{C}$ under a nitrogen atmosphere was treated with $\mathrm{NaH}$ (43 mg of a $55 \%$ dispersion in mineral oil, 0.98 $\mathrm{mmol}$ ). The ensuing mixture was stirred at $20{ }^{\circ} \mathrm{C}$ for $2 \mathrm{~h}$ and then quenched with $\mathrm{NH}_{4} \mathrm{Cl}(5 \mathrm{~mL}$ of a saturated aqueous solution), diluted with water $(20 \mathrm{~mL})$, and extracted with dichloromethane $(3 \times 20 \mathrm{~mL})$. The combined organic phases were washed with brine $(1 \times 50 \mathrm{~mL})$ before being dried $\left(\mathrm{Na}_{2} \mathrm{SO}_{4}\right)$, filtered, and concentrated under reduced pressure. The residue thus obtained was subjected to flash chromatography (silica, 10:1 v/v hexane/ethyl acetate elution) to afford two fractions, $\mathrm{A}$ and $\mathrm{B}$.

Concentration of fraction A $\left(R_{f}=0.5\right.$ in $4: 1 \mathrm{v} / \mathrm{v}$ hexane/ethyl acetate) afforded compound $\mathbf{1 3}(20 \mathrm{mg}, 15 \%)$ as a clear, colorless oil. ${ }^{1} \mathrm{H}$ NMR $\left(400 \mathrm{MHz}, \mathrm{CDCl}_{3}\right) \delta 11.10(\mathrm{~s}, 1 \mathrm{H}), 6.44(\mathrm{~d}, J=2.4 \mathrm{~Hz}$, $1 \mathrm{H}), 6.29(\mathrm{~d}, J=2.4 \mathrm{~Hz}, 1 \mathrm{H}), 6.16(\mathrm{~s}, 1 \mathrm{H}), 5.79(\mathrm{~m}, 1 \mathrm{H}), 5.06-4.94$ (complex m, 2H), $3.85(\mathrm{~s}, 3 \mathrm{H}), 2.49(\mathrm{t}, J=7.6 \mathrm{~Hz}, 2 \mathrm{H}), 2.09(\mathrm{~m}, 2 \mathrm{H})$, $1.70(\mathrm{~m}, 2 \mathrm{H}), 1.46(\mathrm{~m}, 2 \mathrm{H}) ;{ }^{13} \mathrm{C}$ NMR $\left(100 \mathrm{MHz}, \mathrm{CDCl}_{3}\right) \delta 166.9$, $166.6,163.8,157.9,139.5,138.4,115.0,104.1,101.2,100.3,100.1$, $55.8,33.5,33.3,28.3,26.4$; IR $\nu_{\max } 3081,2932,2857,1683,1646$, 1621, 1572, 1511, 1380, 1237, 1163, 1069, $846 \mathrm{~cm}^{-1}$; MS (ESI, +ve) $m / z 297\left[(\mathrm{M}+\mathrm{Na})^{+}, 100 \%\right], 275\left[(\mathrm{M}+\mathrm{H})^{+}, 2\right] ; \operatorname{HRMS}(\mathrm{M}+\mathrm{H})^{+}$ calcd for $\mathrm{C}_{16} \mathrm{H}_{19} \mathrm{O}_{4}, 275.1283$; found, 275.1283 .

Concentration of fraction B $\left(R_{f}=0.2\right.$ in $4: 1 \mathrm{v} / \mathrm{v}$ hexane/ethyl acetate) afforded a white, amorphous solid. Crystallization of this material (hexane/ethyl acetate) then gave compound 14 (50 mg, 35\%) as a white, crystalline solid. mp $94-96{ }^{\circ} \mathrm{C} .{ }^{1} \mathrm{H}$ NMR $(400 \mathrm{MHz}$, $\left.\mathrm{CDCl}_{3}\right) \delta 11.13(\mathrm{~s}, 1 \mathrm{H}), 6.36(\mathrm{~d}, J=2.4 \mathrm{~Hz}, 1 \mathrm{H}), 6.29(\mathrm{~d}, J=2.4 \mathrm{~Hz}$, $1 \mathrm{H}), 5.80(\mathrm{~m}, 1 \mathrm{H}), 5.03-4.95($ complex m, $2 \mathrm{H}), 3.82(\mathrm{~s}, 3 \mathrm{H}), 3.16(\mathrm{~d}$, $J=16.1 \mathrm{~Hz}, 1 \mathrm{H}), 3.00(\mathrm{~d}, J=16.1 \mathrm{~Hz}, 1 \mathrm{H}), 2.09(\mathrm{~m}, 2 \mathrm{H}), 1.95(\mathrm{~m}$,
$2 \mathrm{H}$ ), 1.68-1.44 (complex m, 4H) (signal due to carboxylic acid group proton not observed); ${ }^{13} \mathrm{C}$ NMR $\left(100 \mathrm{MHz}, \mathrm{CDCl}_{3}\right) \delta 169.3,166.3$, 164.6, 139.1, 138.5, 115.0, 107.6, 104.6, 101.3, 99.7, 55.7, 40.8, 36.8, 33.6, 28.8, 22.9; IR $\nu_{\max } 3377,2933,1645,1629,1584,1505,1439$, $1362,1307,1205,1160,1064,912 \mathrm{~cm}^{-1}$; MS (ESI, +ve) $\mathrm{m} / z 315[(\mathrm{M}$ $\left.+\mathrm{Na})^{+}, 100 \%\right], 293\left[(\mathrm{M}+\mathrm{H})^{+}, 15\right]$; HRMS $(\mathrm{M}+\mathrm{H})^{+}$calcd for $\mathrm{C}_{16} \mathrm{H}_{21} \mathrm{O}_{5}$, 293.1389; found, 293.1388.

Method B. A magnetically stirred mixture of compound $10(54 \mathrm{mg}$, $0.16 \mathrm{mmol})$, ethylene glycol $(198 \mathrm{mg}, 3.2 \mathrm{mmol})$, and $p-\mathrm{TsOH} \cdot \mathrm{H}_{2} \mathrm{O}$ $(6 \mathrm{mg}, 0.03 \mathrm{mmol})$ in toluene $(5 \mathrm{~mL})$ was heated at reflux for $16 \mathrm{~h}$ in an apparatus fitted with a Dean-Stark trap topped by a Liebig condenser. The cooled reaction mixture was partitioned between dichloromethane $(20 \mathrm{~mL})$ and brine/water $(20 \mathrm{~mL}$ of a $1: 1 \mathrm{v} / \mathrm{v}$ mixture). The separated aqueous phase was extracted with dichloromethane $(3 \times 20 \mathrm{~mL})$, and the combined organic phases were washed with brine $(1 \times 50 \mathrm{~mL})$ before being dried $\left(\mathrm{Na}_{2} \mathrm{SO}_{4}\right)$, filtered, and concentrated under reduced pressure. The residue thus obtained was subjected to flash chromatography (silica, $8: 1 \mathrm{v} / \mathrm{v}$ hexane/ethyl acetate elution) affording, after concentration of the appropriate fractions, compound 13 (37 mg, 82\%) as a clear, colorless oil. This material was identical, in all respects, with that obtained by Method A.

Compound 14. A magnetically stirred solution of compound 10 (382 mg, $1.15 \mathrm{mmol})$ in $\mathrm{THF} / \mathrm{H}_{2} \mathrm{O}(50 \mathrm{~mL}$ of a $1: 1 \mathrm{v} / \mathrm{v}$ mixture $)$ was treated with $\mathrm{KOH}(322 \mathrm{mg}, 5.75 \mathrm{mmol})$, and the ensuing yellow solution was heated at reflux for $16 \mathrm{~h}$. The cooled reaction mixture was concentrated under reduced pressure, and the residue was acidified, using $\mathrm{HCl}$ ( $2 \mathrm{M}$ aqueous solution), to $\mathrm{pH} 1$. The suspension thus formed was diluted with brine $(50 \mathrm{~mL})$ and then extracted with ethyl acetate $(3 \times 50 \mathrm{~mL})$. The combined organic phases were dried $\left(\mathrm{MgSO}_{4}\right)$, filtered, and concentrated under reduced pressure to give a light yellow oil. The residue so obtained was subjected to flash chromatography (silica, 2:1:0.01 v/v/v hexane/ethyl acetate/acetic acid elution) to afford, after concentration of the relevant fractions $\left(R_{f}\right.$ $=0.2$ in $4: 1 \mathrm{v} / \mathrm{v}$ hexane/ethyl acetate), compound 14 (322 mg, 96\%) as a white, amorphous solid. This material was identical, in all respects, with that obtained by Method A as detailed immediately above.

Compound 12. A magnetically stirred solution of $\mathrm{P}(\text { fur })_{3}(164 \mathrm{mg}$, $0.70 \mathrm{mmol})$ in benzene $(2 \mathrm{~mL})$ was treated with DIAD $(175 \mu \mathrm{L}, 0.88$ $\mathrm{mmol}$ ), the ensuing yellow solution was stirred at $20{ }^{\circ} \mathrm{C}$ for $10 \mathrm{~min}$, and then $(S)-(+)-4$-penten-2-ol $(32 \mathrm{mg}, 0.37 \mathrm{mmol}$ ) was added dropwise. The resulting mixture was stirred at $20^{\circ} \mathrm{C}$ for $5 \mathrm{~min}$ and was then treated, dropwise, with a solution of acid $14(103 \mathrm{mg}, 0.35$ mmol) in benzene $(7 \mathrm{~mL})$. The ensuing mixture was stirred at $30{ }^{\circ} \mathrm{C}$ for $16 \mathrm{~h}$ and then concentrated under reduced pressure. The lightyellow oil thus obtained was subjected to flash chromatography (silica, $30: 1 \rightarrow 15: 1 \mathrm{v} / \mathrm{v}$ hexane/ethyl acetate gradient elution) to afford, after concentration of the relevant fractions $\left(R_{f}=0.6\right.$ in $4: 1 \mathrm{v} / \mathrm{v}$ hexane/ ethyl acetate), compound $\mathbf{1 2}$ (98 $\mathrm{mg}, 78 \%)$ as a clear, colorless oil. $[\alpha]_{\mathrm{D}}^{20}-155\left(c 0.6, \mathrm{CHCl}_{3}\right) .{ }^{1} \mathrm{H}$ NMR $\left(400 \mathrm{MHz}, \mathrm{CDCl}_{3}\right) \delta 11.72(\mathrm{~s}$, $1 \mathrm{H}), 6.40(\mathrm{~d}, J=2.3 \mathrm{~Hz}, 1 \mathrm{H}), 6.21(\mathrm{~d}, J=2.3 \mathrm{~Hz}, 1 \mathrm{H}), 5.75(\mathrm{~m}, 2 \mathrm{H})$, $5.25(\mathrm{~m}, 1 \mathrm{H}), 5.14-5.09$ (complex m, 2H), 4.99-4.91 (complex m, $2 \mathrm{H}), 4.00(\mathrm{~d}, J=17.2 \mathrm{~Hz}, 1 \mathrm{H}), 3.80(\mathrm{~d}, J=17.2 \mathrm{~Hz}, 1 \mathrm{H}), 3.76(\mathrm{~s}, 3 \mathrm{H})$, $2.47-2.30($ complex m, 4H), $2.02(\mathrm{~m}, 2 \mathrm{H}), 1.57(\mathrm{~m}, 2 \mathrm{H}), 1.35(\mathrm{~m}$, $2 \mathrm{H}), 1.28(\mathrm{~d}, J=6.3 \mathrm{~Hz}, 3 \mathrm{H}) ;{ }^{13} \mathrm{C}$ NMR $\left(100 \mathrm{MHz}, \mathrm{CDCl}_{3}\right) \delta 207.4$, $170.2,165.8,164.0,139.0,138.5,133.4,118.4,114.7,112.8,105.8$, $100.1,72.2,55.5,51.4,41.7,40.2,33.6,28.5,23.1,19.5$; IR $\nu_{\max } 3077$, 2978, 2935, 2853,1716, 1646, 1616, 1578, 1430, 1356,1322, 1303, 1257, 1204, 1160,1047, $914 \mathrm{~cm}^{-1}$; MS (ESI, +ve) $\mathrm{m} / z 383[(\mathrm{M}+$ $\left.\mathrm{Na})^{+}, 100 \%\right], 361\left[(\mathrm{M}+\mathrm{H})^{+}, 16\right]$; HRMS $(\mathrm{M}+\mathrm{H})^{+}$calcd for $\mathrm{C}_{21} \mathrm{H}_{29} \mathrm{O}_{5}, 361.2015$; found, 361.2016.

Compound 1. A magnetically stirred solution of diene $\mathbf{1 2}(98 \mathrm{mg}$ $0.27 \mathrm{mmol})$ in dichloromethane $(150 \mathrm{~mL}$, dry and degassed with argon) maintained at $20^{\circ} \mathrm{C}$ under an argon atmosphere was treated, in one portion, with Grubbs' II catalyst $(23 \mathrm{mg}, 0.027 \mathrm{mmol})$. The resulting brown solution was heated at reflux for $24 \mathrm{~h}$, and then another portion of the Grubbs' II catalyst $(23 \mathrm{mg}, 0.027 \mathrm{mmol}$ ) was added. After another $24 \mathrm{~h}$, the reaction mixture was concentrated under reduced pressure, and the residue thus obtained subjected to flash chromatography (silica, 15:1 v/v hexane/ethyl acetate elution). Concentration of the relevant fractions $\left(R_{f}=0.5\right.$ in $4: 1 \mathrm{v} / \mathrm{v}$ hexane/ 
ethyl acetate) gave a white solid that upon recrystallization (hexane/ dichloromethane) afforded compound 1 (74 $\mathrm{mg}, 83 \%)$ as white needles. mp $102-103{ }^{\circ} \mathrm{C} .[\alpha]_{\mathrm{D}}^{20}+40\left(c 0.6, \mathrm{CHCl}_{3}\right)$. All of the NMR, IR, and MS spectral data recorded on this compound were essentially identical to those derived from compound ent-1 (see below).

Compound ent-12. A magnetically stirred solution of $\mathrm{P}(\mathrm{fur})_{3}$ $(171 \mathrm{mg}, 0.74 \mathrm{mmol})$ in benzene $(3 \mathrm{~mL})$ was treated, dropwise, with DIAD $(182 \mu \mathrm{L}, 0.91 \mathrm{mmol})$, the resulting yellow solution was stirred at $20{ }^{\circ} \mathrm{C}$ for $10 \mathrm{~min}$, and then $(R)-(-)-4$-penten-2-ol $(33 \mathrm{mg}, 0.38$ $\mathrm{mmol}$ ) was added. The ensuing mixture was stirred at $20^{\circ} \mathrm{C}$ for $5 \mathrm{~min}$ and then treated, dropwise, with a solution of acid $\mathbf{1 4}$ (106 mg, 0.36 mmol) in benzene $(7 \mathrm{~mL})$. The mixture thus obtained was stirred at $30{ }^{\circ} \mathrm{C}$ for $16 \mathrm{~h}$ and then concentrated under reduced pressure. The resulting light-yellow oil was subjected to flash chromatography (silica, $30: 1 \rightarrow 15: 1 \mathrm{v} / \mathrm{v}$ hexane/ethyl acetate gradient elution) to afford, after concentration of the relevant fractions $\left(R_{f}=0.6\right.$ in $4: 1 \mathrm{v} / \mathrm{v}$ hexane/ ethyl acetate), diene ent-12 (120 mg, 92\%) as a clear, colorless oil. $[\alpha]_{\mathrm{D}}^{20}+158\left(c 0.6, \mathrm{CHCl}_{3}\right)$. All of the NMR, IR, and MS spectral data recorded on this compound were identical to those derived from compound 12 (see above).

Compound ent-1. A magnetically stirred solution of diene ent-12 (34 $\mathrm{mg}, 0.094 \mathrm{mmol})$ in dichloromethane $(10 \mathrm{~mL}$, dry and degassed with argon) maintained under argon was treated, in one portion, with Grubbs' II catalyst (12 mg, $0.014 \mathrm{mmol}$ ). The ensuing brown solution was heated at reflux for $3 \mathrm{~h}$ and then concentrated under reduced pressure. The brown oil thus obtained was subjected to flash chromatography (silica, 15:1 v/v hexane/ethyl acetate elution). Concentration of the relevant fractions $\left(R_{f}=0.5\right.$ in $4: 1 \mathrm{v} / \mathrm{v}$ hexane/ ethyl acetate) gave a white solid that upon recrystallization (hexane/ dichloromethane) afforded compound ent-1 $(20 \mathrm{mg}, 67 \%)$ as white needles. $\mathrm{mp} 102-103{ }^{\circ} \mathrm{C}$ (lit. $\left.{ }^{2 \mathrm{a}} \mathrm{mp} 160-161{ }^{\circ} \mathrm{C}\right),[\alpha]_{\mathrm{D}}^{20}-42(c 0.6$, $\left.\mathrm{CHCl}_{3}\right)\left\{\right.$ lit. $\left.^{2 \mathrm{a}}[\alpha]_{\mathrm{D}}^{25}-43\left(\mathrm{c} 0.6, \mathrm{CHCl}_{3}\right)\right\} .{ }^{1} \mathrm{H}$ NMR $(400 \mathrm{MHz}$, $\left.\mathrm{CDCl}_{3}\right) \delta$ see Table $1 ;{ }^{13} \mathrm{C}$ NMR $\left(100 \mathrm{MHz}, \mathrm{CDCl}_{3}\right) \delta$ see Table 1 ; IR $\nu_{\max } 2975,2934,2848,1709,1646,1614,1577,1381,1355,1305$, $1257,1220,1203,1161,1112,1042,961 \mathrm{~cm}^{-1}$; MS (ESI, +ve) $\mathrm{m} / z$ $355\left[(\mathrm{M}+\mathrm{Na})^{+}, 100 \%\right], 333\left[(\mathrm{M}+\mathrm{H})^{+}, 19\right]$; HRMS $(\mathrm{M}+\mathrm{Na})^{+}$ calcd for $\mathrm{C}_{19} \mathrm{H}_{24} \mathrm{NaO}_{5}, 355.1521$; found, 355.1521 .

Crystallographic Studies. Crystallographic Data for Compound ent-1. $\mathrm{C}_{19} \mathrm{H}_{24} \mathrm{O}_{5}, \mathrm{M}=332.40, T=150 \mathrm{~K}$, orthorhombic, space group $P 2_{1} 2_{1} 2_{1}, Z=4, a=5.1830(1), b=12.7946(2), c=$ 26.0924(3) $\AA ; V=1730.30(5) \AA^{3}, D_{x}=1.276 \mathrm{~g} \mathrm{~cm}^{-3}, 3372$ unique data $\left(2 \theta_{\max }=144.4^{\circ}\right), R=0.033$ [for 3320 reflections with $I>$ $2.0 \sigma(I)] ; R w=0.087$ (all data), $S=1.00$.

Structure Determination. Images were measured on a CCD diffractometer ( $\mathrm{Cu} \mathrm{K} \alpha$, mirror monochromator, $\lambda=1.54184 \AA$ ), and data was extracted using the CrysAlis package. ${ }^{15}$ Structure solution was by direct methods (SIR92). ${ }^{16}$ The structure of compound ent-1 was refined using the CRYSTALS program package. ${ }^{17}$ Atomic coordinates, bond lengths and angles, and displacement parameters for compound ent-1 have been deposited at the Cambridge Crystallographic Data Centre (CCDC no. 1053186). These data can be obtained free of charge via www.ccdc.cam.ac.uk/data_request/cif, by emailing data request@ccdc.cam.ac.uk, or by contacting The Cambridge Crystallographic Data Centre, 12 Union Road, Cambridge CB2 1EZ, UK; fax: +44 1223336033 .

\section{ASSOCIATED CONTENT}

\section{S Supporting Information}

Crystallographic data (CIF); anisotropic displacement ellipsoid plot derived from the single-crystal analysis of compound ent-1; ${ }^{1} \mathrm{H}$ and ${ }^{13} \mathrm{C}$ NMR spectra data for compounds 1, ent-1, 6-10, 12, ent-12, 13, and 14. This material is available free of charge via the Internet at http://pubs.acs.org.

\section{AUTHOR INFORMATION}

\section{Corresponding Author}

*E-mail: Martin.Banwell@anu.edu.au.

\section{Notes}

The authors declare no competing financial interest.

\section{ACKNOWLEDGMENTS}

We thank the Australian Research Council and the Institute of Advanced Studies for financial support. Y.Z. is the grateful recipient of a CSC Ph.D. Scholarship provided by the Government of the People's Republic of China.

\section{REFERENCES}

(1) For useful points of entry into the literature on RALs, see: (a) Winssinger, N.; Barluenga, S. Chem. Commun. 2007, 22. (b) Barluenga, S.; Dakas, P.-Y.; Boulifa, M.; Moulin, E.; Winssinger, N. C. R. Chim. 2008, 11, 1306. (c) Hofmann, T.; Altmann, K.-H. C. R. Chim. 2008, 11, 1318. (d) Bräse, S.; Encinas, A.; Keck, J.; Nising, C. F. Chem. Rev. 2009, 109, 3903. (e) Napolitano, C.; Murphy, P. V. Resorcylic acid lactones. In Natural Lactones and Lactams: Synthesis, Occurrence and Biological Activity; Janecki, T., Ed.; Wiley-VCH: Weinheim, Germany, 2014; Chapter 7. (f) Cookson, R.; Barrett, T. N.; Barrett, A. G. M. Acc. Chem. Res. 2015, 48, 628.

(2) (a) Gao, J.; Radwan, M. M.; León, F.; Dale, O. R.; Husni, A. S.; Wu, Y.; Lupien, S.; Wang, X.; Manly, S. P.; Hill, R. A.; Dugan, F. M.; Cutler, H. G.; Cutler, S. J. J. Nat. Prod. 2013, 76, 824. (b) Gao, J.; Radwan, M. M.; León, F.; Dale, O. R.; Husni, A. S.; Wu, Y.; Lupien, S.; Wang, X.; Manly, S. P.; Hill, R. A.; Dugan, F. M.; Cutler, H. G.; Cutler, S. J. J. Nat. Prod. 2013, 76, 2174.

(3) (a) Lin, A.; Willis, A. C.; Banwell, M. G. Heterocycles 2010, 82, 313. (b) Lin, A.; Willis, A. C.; Banwell, M. G. Tetrahedron Lett. 2010, 51, 1044. (c) Bolte, B.; Basutto, J. A.; Bryan, C. S.; Garson, M. J.; Banwell, M. G.; Ward, J. S. J. Org. Chem. 2015, 80, 460.

(4) Dachavaram, S. S.; Balasaheb, K.; Das, S. Tetrahedron Lett. 2014, $55,5629$.

(5) For some examples of syntheses of RALs that use RCMs for the purposes of constructing the associated 14-membered macrolide, see: (a) Fürstner, A.; Thiel, O. R.; Kindler, N.; Bartkowska, B. J. Org. Chem. 2000, 65, 7990. (b) Garbaccio, R. M.; Stachel, S. J.; Baeschlin, D. K.; Danishefsky, S. J. J. Am. Chem. Soc. 2001, 123, 10903. (c) Moulin, E.; Barluenga, S.; Totzke, F.; Winssinger, N. Chem.-Eur. J. 2006, 12, 8819. (d) Navarro, I.; Basset, J.-F.; Hebbe, S.; Major, S. M.; Werner, T.; Howsham, C.; Bräckow, J.; Barrett, A. G. M. J. Am. Chem. Soc. 2008, 130, 10293. (e) Karthikeyan, G.; Zambaldo, C.; Barluenga, S.; Zoete, V.; Karplus, M.; Winssinger, N. Chem.-Eur. J. 2012, 18, 8978.

(6) Denmark, S. E.; Middleton, D. S. J. Org. Chem. 1998, 63, 1604.

(7) Taber, D. F.; DeMatteo, P. W.; Hassan, R. A. Org. Synth. 2013, 90, 350.

(8) Kulasegaram, S.; Kulawiec, R. J. J. Org. Chem. 1997, 62, 6547.

(9) Danishefsky et al. have shown (ref $5 b$ ) that by using this phosphine in the Mitsunobu reaction then competing lactonization reactions (in our case, leading to the isocoumarin 13) are suppressed.

(10) Swamy, K. C. K.; Kumar, N. N. B.; Balaraman, E.; Kumar, K. V. P. P. Chem. Rev. 2009, 109, 2551.

(11) Scholl, M.; Ding, S.; Lee, C. W.; Grubbs, R. H. Org. Lett. 1999, $1,953$.

(12) Given the nature of the chemistry employed in the Das synthesis (in particular the use of a Mitsunobu esterification reaction), this almost certainly leads to the $S$-enantiomer of neocosmosin A (viz. ent-1) and not the $R$-enantiomer (viz. 1) as illustrated in their publication (ref 4).

(13) Still, W. C.; Kahn, M.; Mitra, A. J. Org. Chem. 1978, 43, 2923.

(14) Pangborn, A. B.; Giardello, M. A.; Grubbs, R. H.; Rosen, R. K.; Timmers, F. J. Organometallics 1996, 15, 1518.

(15) CrysAlis PRO; Agilent Technologies: Santa Clara, CA, 2013.

(16) SIR92: Altomare, A.; Cascarano, G.; Giacovazzo, C.; Guagliardi, A.; Burla, M. C.; Polidori, G.; Camalli, M. J. Appl. Crystallogr. 1994, 27, 435 .

(17) Betteridge, P. W.; Carruthers, J. R.; Cooper, R. I.; Prout, K.; Watkin, D. J. J. Appl. Crystallogr. 2003, 36, 1487. 